Bio - grafia. Escritos sobre la Biología y su Enseñanza. ISSN 2027

Edición Extraordinaria. p.p. 891- 904

Memorias del VIII Encuentro Nacional de Experiencias en Enseñanza de la Biología y la Educación Ambiental. III Congreso Nacional de Investigación en Enseñanza de la Biología.

\title{
ENSEÑANZA SOBRE BIODIVERSIDAD DESDE EL CONOCIMIENTO PEDAGÓGICO DEL CONTENIDO DE UN FUTURO PROFESOR: UN ESTUDIO DE CASO.
}

\author{
Cuellar López Zully
}

\section{Resumen}

La investigación busca desarrollar desde el más importante espacio para un futuro profesor, la práctica pedagógica, el Conocimiento Pedagógico de Contenido (CPC) sobre el concepto de biodiversidad, del estudiante en formación de la Universidad Surcolombiana Neiva-Huila, del programa de Licenciatura en Ciencias Naturales: física, química y biología. EI CPC considerado como una amalgama entre disciplina y pedagogía, permite fortalecer el saber profesional docente y contribuir a resolver la tensión existente entre los que plantean que la disciplina es suficiente para enseñar y los que consideran lo es la pedagogía. Con una investigación cualitativa, interpretativa en el marco del estudio de caso se construyen los CoRes y PaP-eRs para contribuir al conocimiento sobre la enseñanza del concepto de biodiversidad y sobre la formación de futuros profesionales en la educación en ciencias. En la CoRe indagada, el futuro profesor identifica la idea central asociada con la Biodiversidad, el objetivo de su enseñanza, reconoce las probables dificultades conceptuales de sus estudiantes y propone estrategias para abordar satisfactoriamente la clase y su evaluación.

Palabras claves: práctica docente, CPC, biodiversidad.

\section{Abstract}

The research aims to develop from the most important space for a future teacher, teaching practice, the Pedagogical Content Knowledge (CPC) on the concept of biodiversity, student trainees of Neiva, Huila South Colombian University, the Bachelor of Science natural physics, chemistry and biology. CPC considered as an amalgam between discipline and pedagogy strengthens teachers' professional knowledge and help resolve the tension between those who argue that the discipline is sufficient to teach and those who believe it is the pedagogy. With a qualitative, interpretive research as part of the case study CoRes and PaP-ers are constructed to contribute to knowledge about teaching the concept of biodiversity and the training of future professionals in science education. In the CoRe

${ }^{1}$ Universidad Surcolombiana, docente de planta. zully.cuellar@usco.edu.co 
Bio - grafia. Escritos sobre la Biología y su Enseñanza. ISSN 2027

Edición Extraordinaria. p.p. 891- 904

Memorias del VIII Encuentro Nacional de Experiencias en Enseñanza de la Biología y la Educación Ambiental. III Congreso Nacional de Investigación en Enseñanza de la Biología.

inquired into, the future teacher identifies the thrust associated with biodiversity, the aim of his teaching, recognizing the likely conceptual difficulties of students and proposes strategies to successfully address the class and their evaluation.

Keywords: teaching practice, CPC, biodiversity.

\section{Introducción}

La formación de futuros profesores debe abordar tensiones que se mantienen desde hace treinta años, entre los que consideran que la formación de los maestros debe hacerse fundamentada en un alto dominio de las ciencias, y consideran secundario en la formación de maestros el saber de la pedagogía y de la didáctica; y los que consideran que la base fundamental de la enseñanza es la pedagogía y la didáctica como su discurso práctico, y que, el solo dominio de un conocimiento científico o técnico no determina las habilidades, ni destrezas que provee la inteligencia pedagógica a la práctica educativa (Ibarra, 2011).

Al momento de iniciar las practicas pedagógicas y de ejercer la profesión docente la interacción entre el conocimiento del tema de la materia (disciplina) y la pedagogía se hace indispensable. Investigaciones al respecto desde hace aproximadamente dos décadas han producido conocimiento que plasma esta interacción. En este sentido, tenemos el Conocimiento Pedagógico del Contenido (CPC) que permite capturar desarrollar, documentar y representar el CPC de un maestro acerca de un tópico específico, con la intención de que este conocimiento sirva de referencia para mejorar la formación de estudiantes profesores y profesores en ejercicio (Loughran, Gunstone, Berry, Milroy, \& Mulhall, 2001). Las prácticas pedagógicas para el futuro docente se convierten en la oportunidad para desarrollar su CPC de un tópico específico, en este caso trabajar el concepto de biodiversidad, tan indispensable para reconocer nuestro accionar con el medio natural y poder participar en la toma de decisiones que comprometen nuestros recursos naturales. Teniendo en cuenta estas ideas surge como pregunta de investigación: ¿Cómo desde la práctica pedagógica un estudiante en formación puede desarrollar el CPC sobre el concepto de biodiversidad?

La relación complementaria entre pedagogía y disciplina es necesaria fortalecerla a través de las investigaciones, para dar respuestas a las problemáticas presentadas en la educación. Estos procesos se han venido abordando en investigaciones desde hace varias 
Bio - grafia. Escritos sobre la Biología y su Enseñanza. ISSN 2027

Edición Extraordinaria. p.p. 891- 904

Memorias del VIII Encuentro Nacional de Experiencias en Enseñanza de la Biología y la Educación Ambiental. III Congreso Nacional de Investigación en Enseñanza de la Biología.

décadas. En el mundo anglosajón desde los estudios de Shulman (1986) ha venido evolucionando con los trabajos de Grossman $(1990,2005)$ y Magnusson et. al, (1999). En Latinoamérica con los estudios mexicanos de Garritz y Velásquez, (2009), Reyes y Garritz (2006). En Colombia, Valbuena (2007) con los estudios en la universidad pedagógica nacional a futuros docentes en Biología y Candela (2012) sobre Discontinuidad de la materia. Los resultados de dichas investigaciones han permitido nuevas conceptualizaciones en el campo de la educación en Ciencias Naturales y en la formación docente, para mejorar la enseñanza de éstas. Surge entonces, el Conocimiento Pedagógico del Contenido (CPC), como lo conceptualizó inicialmente Shulman (1986):"una especie de amalgama entre materia y pedagogía que constituye una esfera exclusiva de los maestros, su propia forma especial de comprensión profesional" que permite documentar y desarrollar el CPC de un tópico específico y ayude a mitigar la tensión disciplina vs pedagogía.

En esta investigación trabajaremos con el CPC para profesores de Ciencias, propuesto por Magnusson et. al, (1999), teniendo en cuenta que la investigación está en el marco del programa de la Licenciatura en Ciencias Naturales. Este autor propone subcategorías para documentar el contenido del CPC acerca de los criterios para la toma de decisiones curriculares y de instrucción. Las subcategorías son las siguientes:

Orientaciones hacia la enseñanza de la ciencia.

Conocimiento y creencias acerca del currículum de la ciencia.

Conocimiento y creencias acerca de las estrategias de instrucción para la enseñanza de la ciencia.

Conocimiento y creencias acerca de la comprensión de los estudiantes de un tópico específico de la ciencia.

Conocimiento y creencias acerca de la evaluación en la alfabetización científica.

Las subcategorías permiten la planeación, la enseñanza y evaluación del concepto de biodiversidad mediante la construcción de la CoRe y los PaP-eRs para desarrollar el CPC sobre el concepto de biodiversidad en el estudiante en formación. 
Bio - grafia. Escritos sobre la Biología y su Enseñanza. ISSN 2027

Edición Extraordinaria. p.p. 891- 904

Memorias del VIII Encuentro Nacional de Experiencias en Enseñanza de la Biología y la Educación Ambiental. III Congreso Nacional de Investigación en Enseñanza de la Biología.

La Representación del Contenido ( $\mathrm{CoRe}$ ) es un resumen de cómo un profesor enseña un tópico específico y, de las razones por las que lo enseña de esa manera. Así pues, estas representaciones permiten recopilar el CPC del profesor de ciencias acerca de un tópico específico, y suministra algunas intuiciones en las decisiones curriculares y de instrucción que toma el enseñante durante la planificación, actuación y reflexión de su práctica pedagógica. Recoge elementos claves del acto educativo: estudiante, contenidos específicos y práctica del docente (Candela, 2012).

Los Repertorios de experiencias Profesionales y Pedagógicas (PaP-eRs) son relatos narrativos del CPC de los profesores donde se muestra el CPC en la acción. "Desempaca" el pensamiento del profesor alrededor de su CPC, (Garritz et. al, 2006).

\section{Metodología}

Un estudio del tipo interpretativo del problema con carácter cualitativo utilizando el estudio de caso, basado en los relatos narrativos, construye un conocimiento sobre la formación de maestros a partir de la interpretación de quienes la producen y viven. El estudiante profesor seleccionado para la investigación del $8^{\circ}$ semestre del programa de Licenciatura en Ciencias Naturales: Física, Química y Biología, de la Universidad Surcolombiana realizaba su primera práctica pedagógica en el grado $9^{\circ}$ de una institución educativa de Neiva-Huila con 37 estudiantes. Se caracteriza por su alta formación en investigación disciplinar, pertenece al Grupo de "Investigación y Pedagogía en Biodiversidad" GIPB, que tiene como objetivo el estudio de aves, anfibios y reptiles, con el reconocimiento, inventario, comportamiento y distribución de estos grupos taxonómicos en diferentes ecosistemas, en especial en bosque seco tropical.

\section{Selección de los Instrumentos de Recolección y Análisis de Datos}

De acuerdo a investigaciones similares sobre el desarrollo del CPC de un tópico especifico, se tuvo en cuenta dos instrumentos diseñados y validados en investigaciones para tal fin en Loughran et. al (2001): la CoRe y los PaP-eRs. Estos surgen ante la necesidad de rediseñar una metodología que permitiera la identificación y representación de los diferentes elementos del CPC para otros, en el marco teórico del estudio de caso. Los PaP-eRs se construyen con base a los relatos narrativos (RN) producto de las observaciones participantes, videos de las clases y entrevistas, en este texto por cuestiones de espacio no 
Bio - grafia. Escritos sobre la Biología y su Enseñanza. ISSN 2027

Edición Extraordinaria. p.p. 891- 904

Memorias del VIII Encuentro Nacional de Experiencias en Enseñanza de la Biología y la Educación Ambiental. III Congreso Nacional de Investigación en Enseñanza de la Biología.

se reproducen los PaP-eRs. Los RN inician con el desarrollo del CoRe de manera que sean significativos y accesible al lector, útil para la reflexión del CPC en mención (Garritz et al, 2006).

La CoRe inicia su desarrollo con el cuestionario base para la entrevista, aplicado al estudiante profesor, explicitando las ideas centrales que quiere enseñar sobre Biodiversidad y su relación con el CPC. La CoRe recoge los elementos del CPC del docente de ciencias según Magnusson et. al., (1999) de la siguiente manera (ver tabla 1):

Tabla 1. Relación CoRe y subcategorías de Magnusson.

\begin{tabular}{|c|c|c|}
\hline \multirow{6}{*}{ 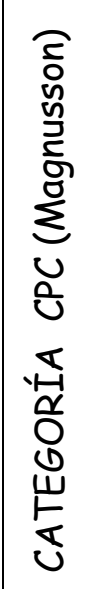 } & Subcategorías & $\begin{array}{l}\text { Preguntas del } \\
\text { cuestionario CoRe. }\end{array}$ \\
\hline & Orientaciones hacia la enseñanza de la ciencia. & 2,5 \\
\hline & $\begin{array}{l}\text { Conocimiento y creencias acerca del currículum de la } \\
\text { ciencia. }\end{array}$ & 1,3 \\
\hline & $\begin{array}{l}\text { Conocimiento y creencias acerca de las estrategias de } \\
\text { instrucción para la enseñanza de la ciencia. }\end{array}$ & 5 \\
\hline & $\begin{array}{l}\text { Conocimiento y creencias acerca de la comprensión de } \\
\text { los estudiantes de un tópico específico de la ciencia. }\end{array}$ & 3 y 4 \\
\hline & $\begin{array}{l}\text { Conocimiento y creencias acerca de la evaluación en } \\
\text { ciencia. }\end{array}$ & 6 \\
\hline
\end{tabular}

El análisis de datos es de forma inductiva, guiados por el marco teórico de la investigación y categorías establecidas. Estas corresponden a las ideas centrales a enseñar del concepto biodiversidad y al CPC. El procesamiento de la información se hará según la propuesta de Strauss y Corbin (2002) la cual consiste en: Lectura de los RN. Organización de datos a través de códigos. Estos representan las dimensiones de las subcategorías de Magnusson para el CPC. Comparación de códigos. Análisis inductivo. El investigador se centra en las áreas de interés (dimensiones de las categorías).Análisis profundo de investigación.

Lo anterior para la construcción de una teoría fundamentada cobre el CPC de un estudiante profesor sobre el tópico Biodiversidad, que surge a partir de la relación estrecha entre la recolección de datos y su análisis.

\section{Resultados y discusión.}


Bio - grafia. Escritos sobre la Biología y su Enseñanza. ISSN 2027

Edición Extraordinaria. p.p. 891- 904

Memorias del VIII Encuentro Nacional de Experiencias en Enseñanza de la Biología y la Educación Ambiental. III Congreso Nacional de Investigación en Enseñanza de la Biología.

Al inicio de la construcción de la CoRe (ver tabla 2) nos encontramos con la idea 1 expresada en el siguiente cuestionario la cual es interrogada con 6 preguntas que permitió el desarrollo del CPC sobre biodiversidad del estudiante profesor.

Tabla 2. CoRe construida con la primera idea.

IDEAS/CONCEPTOS

IMPORTANTES EN CIENCIAS

\section{IDEA 1}

La biodiversidad comprende la variedad de ecosistemas y las diferencias genéticas dentro de cada especie que permiten la combinación de múltiples formas de vida $y$ cuyas mutuas interacciones con el resto del entorno fundamentan el sustento de la vida sobre el planeta.

1. ¿Qué intenta que La biodiversidad depende de la variedad de especies que aprendan los alumnos existen en el planeta tierra y que, por ende es necesario alrededor de esta idea? proteger a todas y cada una de estas para preservar la vida en nuestro planeta. Para entender la biodiversidad hay que comprender conceptos como densidad poblacional, diversidad, abundancia y riqueza, esto a nivel eco sistémico - macro. Si hablamos de especies ya tenemos que comprender la biodiversidad a nivel genético o sea a nivel micro.

2. ¿Por qué es importante que los alumnos sepan esta idea?

Porque la biodiversidad es el diario a vivir de ellos, por ejemplo, si caminas por la calle te encuentras con un perro, gato, iguana, los humanos, zancudos etc. diversas especies con las que interactúas a diario. Es la interacción constante con la naturaleza y porque es necesario desarrollar una mentalidad de conservación de absolutamente todo lo que nos rodea, no solo lo que está en peligro de extinción, conocimiento sobre la variabilidad genética entre las mismas especies (sp), conociendo como cuantificar la diversidad, índice de Shannon - Wiener- $\left(H^{\prime}\right)$ que mide diversidad Alfa o sea la diversidad intrínseca de una comunidad.

3. ¿Cuáles son las dificultades/limitaciones relacionadas con
El desconocimiento total de esta idea por parte de los profesores y estudiantes.

la Otra limitante propias de la ciencias naturales son los 
Bio - grafia. Escritos sobre la Biología y su Enseñanza. ISSN 2027

Edición Extraordinaria. p.p. 891- 904

Memorias del VIII Encuentro Nacional de Experiencias en Enseñanza de la Biología y la Educación Ambiental. III Congreso Nacional de Investigación en Enseñanza de la Biología.

\begin{tabular}{|c|c|}
\hline enseñanza de esta idea? & $\begin{array}{l}\text { niveles macro y micro para entender conceptos como la } \\
\text { biodiversidad esta se ve a nivel macro en los ecosistemas y } \\
\text { especies pero no a nivel micro en los genes. Además para } \\
\text { detectarla se requieren años de observación, el presente da } \\
\text { una imagen estática de las transformaciones de los seres } \\
\text { vivos, para los estudiantes es difícil entender que el ser vivo } \\
\text { está en constante movimiento y más si no se aprecian sus } \\
\text { cambios en el corto tiempo de vida que vivimos, para ellos } \\
\text { pareciera que todo siempre ha sido como lo ven en el } \\
\text { presente. }\end{array}$ \\
\hline $\begin{array}{l}\text { 4. ¿Qué conocimientos } \\
\text { acerca del pensamiento de } \\
\text { los alumnos influyen en } \mathrm{su}^{2} \\
\text { enseñanza de esta idea? }\end{array}$ & $\begin{array}{l}\text { Confusión conceptual que tiene la mayoría de los } \\
\text { estudiantes al pensar que BIODIVERSIDAD (diversidad } \\
\text { biológica) con DIVERSIDAD. La diversidad corresponde } \\
\text { variedad de un solo grupo taxonómico, mientras que la } \\
\text { BIODIVERSIDAD corresponde a la variedad taxonómica } \\
\text { del mundo. } \\
\text { Los estudiantes tienden a considerar que entre más } \\
\text { individuos, (mismasp) más diversidad, lo que les hace pensar } \\
\text { que la abundancia es directamente proporcional a la } \\
\text { diversidad. }\end{array}$ \\
\hline $\begin{array}{l}\text { 5. ¿Cuáles procedimientos } \\
\text { de enseñanza empleará? (y } \\
\text { las razones particulares de } \\
\text { su uso con esta idea). }\end{array}$ & $\begin{array}{l}\text { Para desarrollar esta idea se propone un enfoque didáctico } \\
\text { orientado en el marco de investigación dirigida, que consiste } \\
\text { en: } \\
\text { Transformar el pensamiento y/o la parte conceptual de los } \\
\text { estudiantes y también la parte procedimental y actitudinal, } \\
\text { por lo que hay que ubicar a los estudiantes en un contexto } \\
\text { similar o que emule lo que hace un científico, con el objetivo } \\
\text { de que los estudiantes hagan cosas que son totalmente } \\
\text { diferentes a lo cotidiano, a lo que hace un alumno } \\
\text { tradicionalmente. Es en la parte actitudinal y } \\
\text { procedimental; el cómo hacen y cómo toman las clases, } \\
\text { donde se hace una analogía, de que el profesor debe ser }\end{array}$ \\
\hline
\end{tabular}

\footnotetext{
${ }^{2}$ La pregunta 4 se hace desde la experiencia del docente y desde la experiencia o investigaciones de otro, o sea la literatura.
} 
Bio - grafia. Escritos sobre la Biología y su Enseñanza. ISSN 2027

Edición Extraordinaria. p.p. 891- 904

Memorias del VIII Encuentro Nacional de Experiencias en Enseñanza de la Biología y la Educación Ambiental. III Congreso Nacional de Investigación en Enseñanza de la Biología.

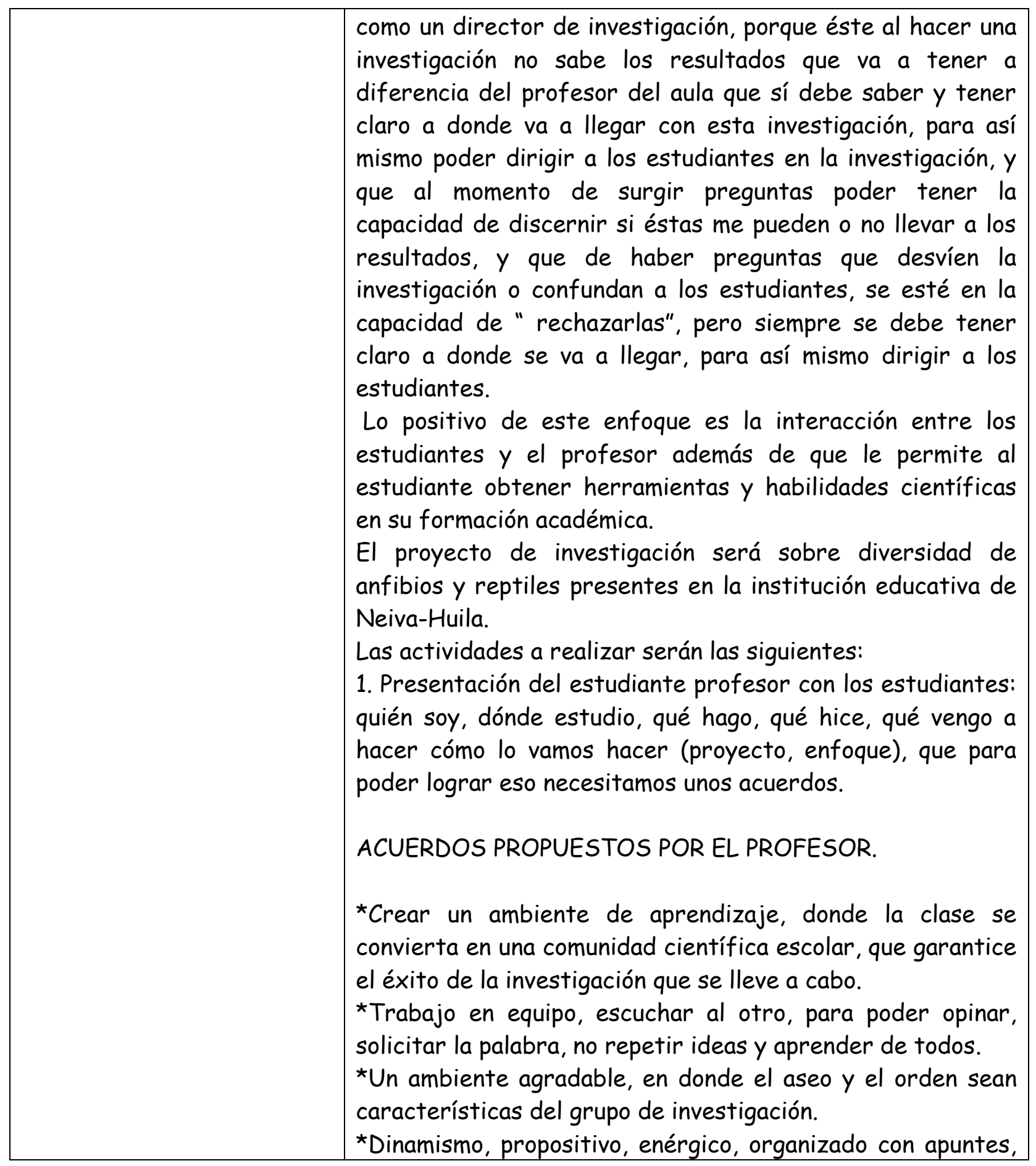


Bio - grafia. Escritos sobre la Biología y su Enseñanza. ISSN 2027

Edición Extraordinaria. p.p. 891- 904

Memorias del VIII Encuentro Nacional de Experiencias en Enseñanza de la Biología y la Educación Ambiental. III Congreso Nacional de Investigación en Enseñanza de la Biología.

líder.

*Sugerencias de los estudiantes investigadores.

2. El docente hará una charla con la importancia de la biodiversidad, para motivar a los estudiantes a protegerla empezando por su colegio y la importancia de conocer los recursos bióticos para entender la biodiversidad.

3. Se procederá a escoger con que grupo taxonómico trabajar (herpetos) para así realizar una introducción acerca del estudio en la herpeto fauna y generalidades sobre estos organismos para centrar a los estudiantes en el grupo faunístico por medio de la Presentación "Conociendo a los herpetos".

4. Taller: Introducción al método científico: esta actividad se desarrollara en paralelo con la elaboración del anteproyecto de investigación, con estas actividades los estudiantes aprenderán:

-. Conocer y comprender el método de investigación que se usa en ciencia.

-. Utilizar el método científico para llegar a comprender diferentes fenómenos de la naturaleza.

-. Desarrollar habilidades científicas: preguntarse, plantear hipótesis, indagar, recoger información y sacar conclusiones, para que los estudiantes puedan explicar de forma sencilla cómo y por qué suceden esos fenómenos y tengan una herramienta útil para llevar a cabo investigaciones.

- Para cada paso del método científico hay unas actividades, para facilitar la construcción de lo explicado en clase.

5. Planeación del anteproyecto de investigación dirigida por el docente con aportes de los estudiantes (esto se elaborará con las actividades recogidas del numeral 4.

6. a Salida de campo, para recoger la información y realizar todo el proceso respectivo de análisis y emitir conclusiones; 
Bio - grafia. Escritos sobre la Biología y su Enseñanza. ISSN 2027

Edición Extraordinaria. p.p. 891- 904

Memorias del VIII Encuentro Nacional de Experiencias en Enseñanza de la Biología y la Educación Ambiental. III Congreso Nacional de Investigación en Enseñanza de la Biología.

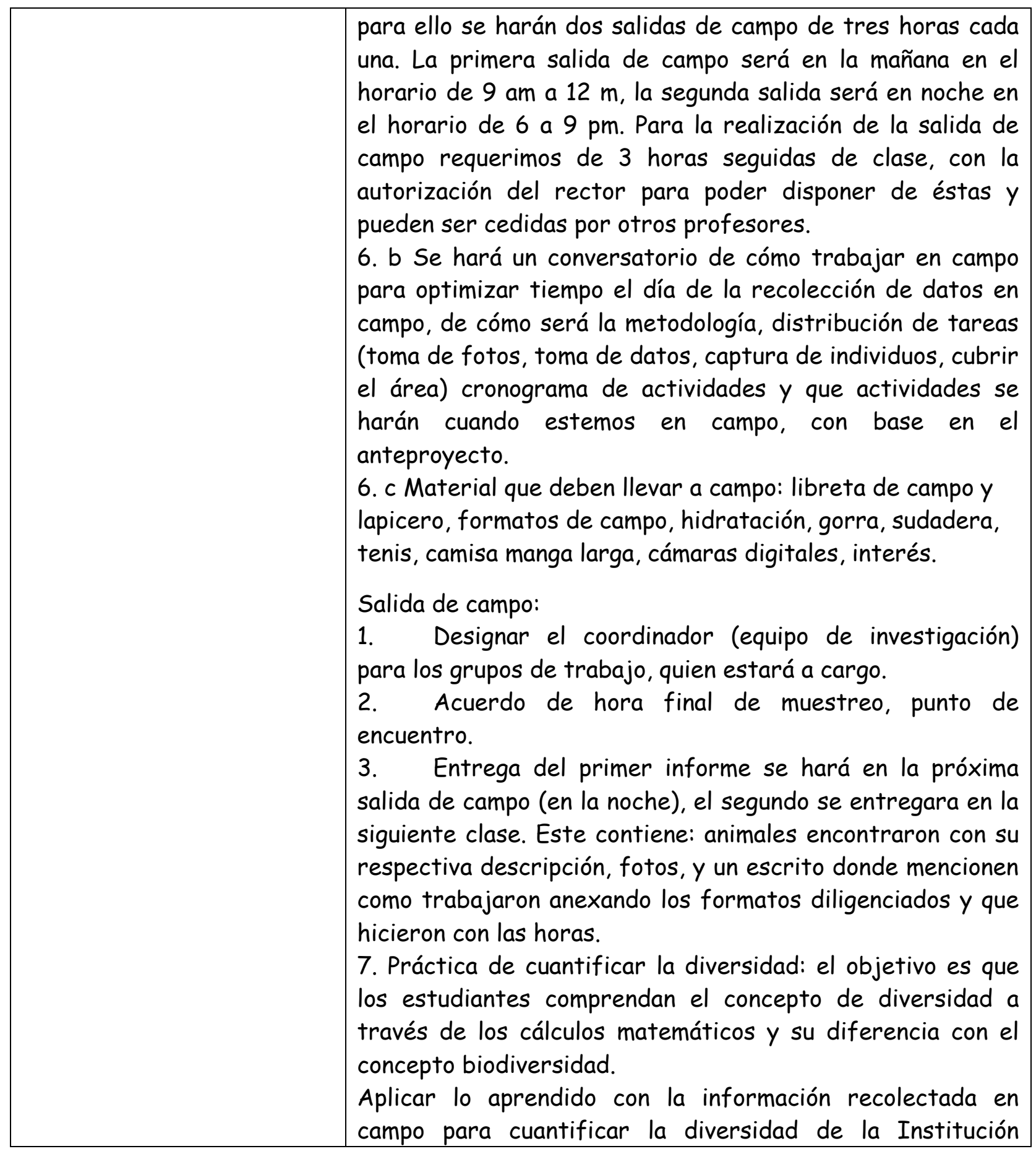


Bio - grafia. Escritos sobre la Biología y su Enseñanza. ISSN 2027

Edición Extraordinaria. p.p. 891- 904

Memorias del VIII Encuentro Nacional de Experiencias en Enseñanza de la Biología y la Educación Ambiental. III Congreso Nacional de Investigación en Enseñanza de la Biología.

\begin{tabular}{|c|c|}
\hline & $\begin{array}{l}\text { educativa, según el índice de Shannon-Wiener. } \\
\text { 8. Se llevaran a cabo lecturas de las especies encontradas } \\
\text { en la salida, dependiendo, de la diversidad. Por grupo o } \\
\text { individualmente exposiciones de una especie en particular } \\
\text { con temas relacionados con ecosistemas que frecuentan } \\
\text { (para entender también que hay diversidad eco sistémicos), } \\
\text { características morfológicas, grado de vulnerabilidad y } \\
\text { nombre científico. Esto con la intención de que los } \\
\text { estudiantes se apropien del conocimiento relacionado con la } \\
\text { herpetofauna existente en su centro de estudios. } \\
\text { 9. Explicación sobre taxonomía, con el objetivo de que los } \\
\text { estudiantes aprendan a identificar las especies que } \\
\text { encontraron en la institución, además de identificar } \\
\text { especies vulnerables, endémicas. } \\
\text { Realización de un taller para dialogar sobre el papel de la } \\
\text { herpetofauna en el ecosistema y elaboración de pautas para } \\
\text { conservar la fauna en el colegio. } \\
\text { 10. Taller de variabilidad genética con especies encontradas } \\
\text { en mis investigaciones. } \\
\text { 11. Elaboración de pósteres con el registro de las especies } \\
\text { encontradas y sugerencias para su conservación en la } \\
\text { institución educativa. } \\
\text { 12. Socialización con la comunidad estudiantil. }\end{array}$ \\
\hline $\begin{array}{l}\text { 6. ¿Qué formas específicas } \\
\text { de evaluación del } \\
\text { entendimiento o de la } \\
\text { confusión de los alumnos } \\
\text { empleara alrededor de esta } \\
\text { idea? }\end{array}$ & $\begin{array}{l}\text { Se evaluará la parte actitudinal que tiene que ver con la } \\
\text { forma de trabajar en grupo, el interés que muestren. En lo } \\
\text { procedimental se tendrá en cuenta la forma de trabajar en } \\
\text { campo, la toma y organización de datos, realización de } \\
\text { cálculos y en lo conceptual sobre: diversidad, abundancia, } \\
\text { índice de Shannon-Wiener. } \\
\text { Mediante el informe de la práctica de campo, además de } \\
\text { entrega periódica de informes de avances que se } \\
\text { presentarán en grupos de trabajo. Por ultimo una evaluación } \\
\text { acumulativa de conceptos básicos que irán aprendiendo a } \\
\text { medida que transcurre la investigación. }\end{array}$ \\
\hline
\end{tabular}


Bio - grafia. Escritos sobre la Biología y su Enseñanza. ISSN 2027

Edición Extraordinaria. p.p. 891- 904

Memorias del VIII Encuentro Nacional de Experiencias en Enseñanza de la Biología y la Educación Ambiental. III Congreso Nacional de Investigación en Enseñanza de la Biología.

A pesar de la que el estudiante profesor es novato, su experiencia como investigador en diversidad animal, le permitió tener claridad sobre la idea principal del concepto biodiversidad y de las categorías de Magnusson las que tenían que ver con: orientaciones hacia la enseñanza de la ciencia y el conocimiento y creencias acerca de la comprensión de los estudiantes de un tópico específico de la ciencia. Con relación a la categoría del conocimiento y creencias acerca de las estrategias de instrucción para la enseñanza de la ciencia, el profesor logra recoger su experiencia como investigador en inventarios de Herpetos para relacionar con el conocimiento pedagógico - didáctico al llevar al aula una propuesta de investigación a los estudiantes. Logra no simular una investigación, sino realizar con los estudiantes el inventario, mediante la toma de muestras, recolección de información, caracterización y determinación del índice de Shannon-Wiener. Su poca experiencia como profesor se aprecia más en el desarrollo de las categorías: conocimiento y creencias acerca del currículum de la ciencia y conocimiento y creencias acerca de la evaluación en ciencia, las cuales se van desarrollando durante la puesta en práctica de la CoRe

\section{Conclusiones}

En la CoRe indagada, el futuro profesor identifica la idea central asociada con la Biodiversidad, el objetivo de su enseñanza, reconoce las probables dificultades conceptuales de sus estudiantes y propone estrategias para abordar satisfactoriamente la clase y su evaluación.

El estudiante profesor concibe la biodiversidad desde un aspecto taxonómico, ecosistémico, genético, más que desde aspectos evolutivos. Por lo detectado en las representaciones de contenido la mayor dificultad para los estudiantes es comprender la diversidad no como cantidad, sino como variedad. Esto se logra resolver con el conocimiento y creencias acerca de las estrategias de instrucción que tiene el estudiante profesor, adquirido en su experiencia como investigador disciplinar, sin embargo en el mismo desarrollo de la CoRe, se hace evidente que no es suficiente el conocimiento disciplinar, es necesaria la reflexión de cómo se presenta ante los estudiantes para su aprendizaje, en este caso sobre biodiversidad.

Esto es el inicio del desarrollo de su CPC que hace al profesor productor de un conocimiento sobre la enseñanza y aprendizaje de las Ciencias Naturales y lo prepara para las eventualidades del aula. 
Bio - grafia. Escritos sobre la Biología y su Enseñanza. ISSN 2027

Edición Extraordinaria. p.p. 891- 904

Memorias del VIII Encuentro Nacional de Experiencias en Enseñanza de la Biología y la Educación Ambiental. III Congreso Nacional de Investigación en Enseñanza de la Biología.

\section{Referentes Bibliográficos}

Candela, B. (2012). La captura, la documentación y la representación del CPC de un profesor experimentado y "ejemplar" acerca del núcleo conceptual de la discontinuidad de la materia. (Tesis de maestría). Universidad del Valle-Colombia.

Garritz y Velasco. (2006). El conocimiento pedagógico de la estructura corpuscular de la materia. Educación en Química, 17, 236-263.

Garritz y Velázquez (Abril, 2009). Biotechnology pedagogical knowledge through Mortimer's conceptual profile. NARST Annual International Conference, USA. Recuperado de: http://garritz.com/andoni_garritz_ruiz/documentos/73-Garritz-Velazquez-BT-PCKProceedings-NARST-2009-FINAL.pdf.

Grossman, P. (1990). The making of a teacher: Teacher knowledge and teacher education. Nueva York: Teachers College Press.

Grossman, P., Wilson, S. y Shulman, L. (2005). Profesores de sustancia: el conocimiento de la materia para la enseñanza. Profesorado. Revista de currículum y formación del profesorado, 9(2).

Ibarra. (2011). Saber pedagógico y saber disciplinar iconvergencia o divergencia? Paidea Surcolombiana, 15, 90-98.

Loughran, J. J., Mulhall, P., and Berry, A. (2004). In search of pedagogical content knowledge in science: Developing ways of articulating and documenting professional practice. Journal of Research in Science Teaching, 41(4), 370-391.

Loughran, Milroy, Berry, Gunstone y Mulhall. (2001). Documenting Science Teachers' Pedagogical Content Knowledge Through PaP-eRs. Research in Science Education. 31, 289307.

Magnusson, S., Krajcik, J. y Borko, H. (1999). Nature, source, and development of pedagogical content knowledge for science teaching, en: J. Gess-Newsome y G. Lederman (eds), PCK and Science Education (pp. 95-132). Holanda.

Reyes-C., F. y Garritz, A. (2006). Conocimiento pedagógico del concepto de 'reacción química' en profesores universitarios mexicanos. Educación en Química, 11 (3), 1175-1205. 
Bio - grafia. Escritos sobre la Biología y su Enseñanza. ISSN 2027

Edición Extraordinaria. p.p. 891- 904

Memorias del VIII Encuentro Nacional de Experiencias en Enseñanza de la Biología y la Educación Ambiental. III Congreso Nacional de Investigación en Enseñanza de la Biología.

Shulman, L.S. (1986). Those who understand: knowledge growth in teaching. Educational Researcher, 15, 4-14.

Strauss y Corbin (2002). Bases de la Investigación Cualitativa. Técnicas y Procedimientos para desarrollar la Teoría Fundamentada. Colombia-Universidad de Antioquia.

Valbuena, (2007) El conocimiento didáctico del contenido biológico. Estudio de las concepciones disciplinares y didácticas de futuros docentes de la universidad pedagógica nacional (Colombia). (Tesis doctoral). Universidad de Valencia. Madrid. 